\title{
Cardiac PET/MRI—an update
}

\author{
C. Rischpler ${ }^{1 *}$, S. G. Nekolla ${ }^{2,3}$, G. Heusch ${ }^{4}$, L. Umutlü ${ }^{5}$, T. Rassaff ${ }^{6}$ P. Heusch7, K. Herrmann ${ }^{1}$ and F. Nensa ${ }^{5}$
}

\author{
* Correspondence: \\ christoph.rischpler@uk-essen.de \\ 'Department of Nuclear Medicine, \\ University Hospital Essen, University \\ of Duisburg-Essen, Essen, Germany \\ Full list of author information is \\ available at the end of the article
}

\begin{abstract}
It is now about 8 years since the first whole-body integrated PET/MRI has been installed. First, reports on technical characteristics and system performance were published. Early after, reports on the first use of PET/MRI in oncological patients were released. Interestingly, the first article on the application in cardiology was a review article, which was published before the first original article was put out. Since then, researchers have gained a lot experience with the PET/MRI in various cardiovascular diseases and an increasing number on auspicious indications is appearing. In this review article, we give an overview on technical updates within these last years with potential impact on cardiac imaging and summarize those scenarios where PET/MRI plays a pivotal role in cardiovascular medicine.
\end{abstract}

Keywords: PET/MRI, Cardiovascular, Clinical applications

\section{Introduction}

In 2010, a new hybrid imaging device was installed for the first time for clinical use and raised great expectations: a truly integrated PET/MRI. Shortly thereafter, a first report on its technical characteristics and performance appeared (Delso et al. 2011). Further reports on its initial applications in oncological scenarios were released soon after. Of note, however, the first published manuscript on cardiovascular applications using this machine was a review article (Rischpler et al. 2013). Until the release of this review in 2013, no original article had been published and-not surprisingly-potential cardiovascular applications were only suggested, for a number of reasons: firstly, PET/ MRI is a complex technology to which clinicians, physicists, researchers, and technologists had first to get used to. And this approach is obviously easier in organs as the brain that do not suffer from motion artifacts due to the heart beat and respiration. Secondly, patients with common cardiovascular applications in PET are still quite rare. Last but not least, and this is the experience of the authors, many cardiovascular imaging studies in PET/MRI are driven by specific, prospective research and not by clinical routine. As cardiac MRI has the advantage of a high soft tissue contrast and permits to assess functional parameters of the left and right ventricle with tissue characterization in great detail, PET has the potential of true molecular imaging with targets such as metabolic pathways, receptors, or cell surface markers. Until today, more and more promising applications of PET/MRI in cardiology have been reported and a guideline on hybrid cardiac PET/MRI has been published (Nensa et al. 2018a). The current review aims to give an update on new technical features and the most promising applications in cardiovascular diseases.

(c) The Author(s). 2019 Open Access This article is distributed under the terms of the Creative Commons Attribution 4.0 International License (http://creativecommons.org/licenses/by/4.0/), which permits unrestricted use, distribution, and reproduction in any medium, provided you give appropriate credit to the original author(s) and the source, provide a link to the Creative Commons license, and indicate if changes were made. 


\section{Technical aspects}

\section{General considerations}

Intrinsic technical aspects of MRI technology such as high magnetic fields, quickly switching gradient fields, and radiofrequency signals may disturb photomultiplier tubes and electronics in "conventional" PET or PET/CT scanners. On the other hand, PET detectors in the field of view of MR scanners may deteriorate MR image quality by electromagnetic interferences and inhomogeneities. These issues were the reason why the construction of an integrated PET/MRI was a technically and also financially demanding project of long duration.

Two different constructions of an integrated whole-body PET/MRI are available, the first being a PET/CT connected with a stand-alone MR scanner connected via table (Philips Ingenuity TF PET/MR, GE-PET/CT-MR) (Zaidi et al. 2011). The second approach represents a truly integrated PET/MR system, where a PET detector ring is installed within the MR tube (Siemens Biograph mMR (Delso et al. 2011), GE SIGNA PET/MR (Levin et al. 2016)). The first construction approach circumvents the aforementioned issues of mutual interferences between PET and MRI. For the truly integrated PET/MRI scanners, novel detector technology had to be engineered: Siemens uses so-called avalanche photodiodes (APDs) (i.e., photodetectors made of lutetium oxyorthosilicate crystal which are insensitive to magnetic fields even at high field strengths) (Pichler et al. 2006), whereas in GE's scanner silicon photomultiplier technology (SiPM)-based PET detectors are installed. One advantage of SiPM is the possibility of time-of-flight PET imaging with a temporal resolution of $<400$ ps (Levin et al. 2014). Only these two vendors have truly integrated PET/MRI scanners on the market. Until today, around 100 Siemens Biograph mMR and 70 GE Signa PET/MRIs have been installed worldwide.

A problem which both integrated scanners had to address was the attenuation correction (AC). AC is obligatory for PET imaging in general and particularly for cardiovascular PET. The reasons for this need are that non-AC PET does not allow the assessment of absolute activity values and artifacts may severely hamper PET data, both of which hinder reliable interpretation of PET images.

\section{AC in PET/MRI}

In stand-alone PET, AC was performed by rotating rod sources (e.g., Germanium-68), a very time-consuming matter. In PET/CT, CT data are used to correct for attenuation by making use of the fact that the density of tissue (and thus the attenuation coefficient) can be easily estimated via Hounsfield units. For integrated PET/MRI, different approaches had to be engineered, as there is no MRI sequence that allows to directly estimate the density of the imaged tissue.

\section{Ways to estimate AC-maps in integrated PET/MRI}

The most frequently used approaches to generate AC-maps from MR data rest on templates, segmentation or atlases, and on PET emission data. Briefly, in the segmentation-based algorithm, the AC-map is generated by different tissue classes, each with a certain attenuation coefficient for $511 \mathrm{keV}$ photons (Huang et al. 1981). This method is applied in the Siemens Biograph mMR where water and fat images are 
generated from a Dixon VIBE MR sequence to subsequently define air, lung, fat, and soft tissue in the AC-map (Martinez-Moller et al. 2009; Coombs et al. 1997). This method requires a breath-hold of about $18 \mathrm{~s}$ per bed position, which may cause problems in very sick patients. While there is an alternative approach using a T1-weighted (T1w) turbo spin echo sequence to shorten image acquisition time for AC-map generation, this approach neglects the density differences between fat and soft tissue $\left(0.086 \mathrm{~cm}^{-1}\right.$ vs. $0.096 \mathrm{~cm}^{-1}$ ) (Schulz et al. 2011). Recently, a novel approach to also model bone using an atlas was suggested and has now been implemented into the Siemens mMR (Paulus et al. 2015). The Philips Ingenuity TF utilizes T1w-GE MR images to segment the three different tissue classes air, lung, and soft tissue. In the GE Signa PET/MRI, a multi-station, whole-body, three-dimensional, dual-echo, RF-spoiled gradient recalled echo (SPGR) sequence (LAVA-Flex) is used and images are segmented into air, lung, fat, and soft tissue. While this approach is similar to that of the Siemens Biograph mMR approach, the difference lies in a classification variation for fat and soft tissue (Beyer et al. 2016). The restriction to a certain number of tissue classes may cause variations in the estimations of radiotracer uptake in thoracic tumors in the range of 5-23\% (Martinez-Moller et al. 2009; Schulz et al. 2011; Samarin et al. 2012). For cardiac imaging, however, studies indicate very close agreement between PET/CT and PET/MRI for fludeoxyglucose (FDG) viability imaging (Lau et al. 2017).

The last approach that will be briefly discussed here is the emission-based approach (maximum likelihood estimation of activity and attenuation (MLAA)), where the non-attenuation corrected PET data are utilized to calculate (missing parts of) the $\mu$-map. A major limitation of this approach is photon scatter (Nuyts et al. 1999). This approach must be mentioned as it is used in PET/MRIs to recover parts of the body (particularly parts of shoulders and arms) which are not "seen" by the MR scanner, but are in the (as compared to the MRI) larger field-of-view of the PET scanner and, of course, contribute to attenuation (Nuyts et al. 2012). A limitation of this technique is that tracers that have no or minor uptake in the truncated parts of the body may also be "overlooked" by this method. In the current version of the Siemens Biograph mMR, a novel approach to overcome this limitation has been introduced-the so-called $\mathrm{B}_{0}$ homogenization using gradient enhancement (HUGE) technique (Blumhagen et al. 2014). Briefly, the readout gradient is optimized by local compensation of $B_{0}$ inhomogeneities via gradient enhancement, resulting in a reduction of truncations outside the field-of-view of the MR scanner. First studies indicate that the HUGE method may be more accurate than the previously used MLAA methodology, particularly when using tracers other than FDG (e.g., I-124) (Fig. 1) (Lindemann et al. 2017). The remaining two AC estimation methods have been described elsewhere and-as they are not implemented in current hybrid PET/MR scanners-will not be discussed here further (Rischpler et al. 2013).

Recently, a novel sequence for AC (the so-called CAIPIRINHA (controlled aliasing in parallel imaging results in higher acceleration) accelerated Dixon 3D-VIBE sequence) with higher resolution but identical scan-time (19 s per bed position) has been introduced (Freitag et al. 2017). The potential of this novel high-resolution sequence is to omit other diagnostic sequences with longer acquisition times, thus allowing to shorten the overall scan-time significantly (which represents one of the major disadvantages of PET/MRI in comparison to PET/CT). 


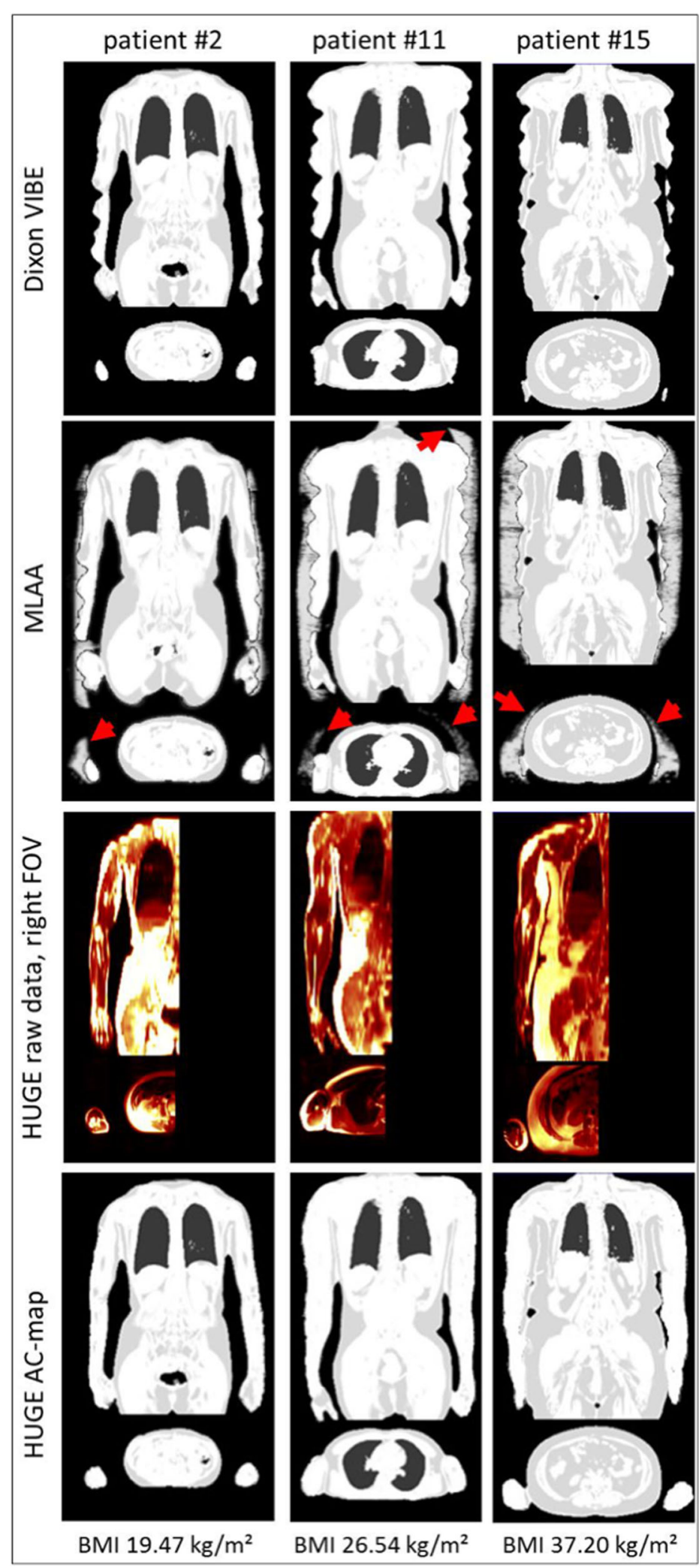

Fig. 1 (See legend on next page.) 
(See figure on previous page.)

Fig. 1 Attenuation correction (AC) map using different methods. AC-maps are shown for the Dixon-VIBE approach (upper row), complemented by MLAA (second row) or HUGE (third row) to recover truncated parts of the body. Red arrows indicate the overestimation around arms and shoulders when applying the MLAA algorithm. Examples of three different patients with increasing body mass index (from left to right) are shown. (Reprinted with permission from Lindemann, M. E., Oehmigen, M., Blumhagen, J. O., Gratz, M. and Quick, H. H. (2017), MR-based truncation and attenuation correction in integrated PET/MR hybrid imaging using HUGE with continuous table motion. Med. Phys., 44: 4559-4572. doi:https://doi.org/10.1002/mp.12449)

\section{Artifacts in AC-map generation by contrast media and implants}

In PET/CT, implanted devices (such as stents, sternal wires, defibrillators, pacemakers, cardiac resynchronization therapy devices) cause artifacts in CT which are translated into the AC-map and ultimately into the attenuation-corrected PET data, where regularly an overestimation of radiotracer uptake is observed. To consider for this issue, non-AC PET data are taken into account, particularly in FDG-PET/CT with the clinical question for infection of, e.g., prosthetic valves to avoid false-positive readings (Jimenez-Ballve et al. 2016). In PET/MRI, however, cardiac (non-magnetic metallic) devices cause a signal that exceeds the actual size of the causing foreign body, resulting in an underestimation of the attenuation and ultimately underestimation of tracer uptake. Obviously, all cardiac devices must be checked with caution if they are approved for a 3T MRI as the magnetic field may cause malfunction or heating (a detailed databank, where most devices' compatibility with MRI may be checked can be found at www.mrisafety.com). However, as many novel cardiac devices enter the market (e.g., left atrial appendage closure devices, transcatheter aortic heart valves, pacing systems, event-recorder), it is often not clear whether or not a device is compatible with MRI and whether or not these devices cause artifacts which translate into PET data and may cause false readings. In a recent study of 20 patients with viability imaging by FDG and ammonia PET/MRI, different AC artifacts were analyzed (Lassen et al. 2017). The authors found artifacts caused by sternal wires or metallic implants in $20 \%$ and $30 \%$ of cases, respectively. Maximum relative differences in the SUVmean ranged from 11 to 196\% in FDG or N-13 PET AC images when correcting for these artifacts (see Fig. 2).

Errors in segmentation are also seen when AC-maps are generated after the application of MR contrast media (Ruhlmann et al. 2016). MR contrast media reduces $\mathrm{T}_{1}$ values which translate into the Dixon VIBE sequence and may cause AC-map errors (Fürst et al. 2012) (see Fig. 3).

\section{Software solutions for image analysis}

There are quite a number of free and commercial software solutions that offer detailed analysis of cardiac PET and MR imaging data. These products often work (semi-)automatically with only limited user interaction. Software applications that truly handle integrated quantitative image analysis of PET and MRI data are still scarce yet, and most software still are only able to handle modality and therefore cannot fully serve the temporal and spatial integrity of simultaneously acquired data. Furthermore, most software focus on oncologic applications and are not specifically designed for the analysis of cardiac imaging data, including the estimation of functional parameters (e.g., end-diastolic and end-systolic volume, ejection fraction), myocardial blood flow, or the generation or 


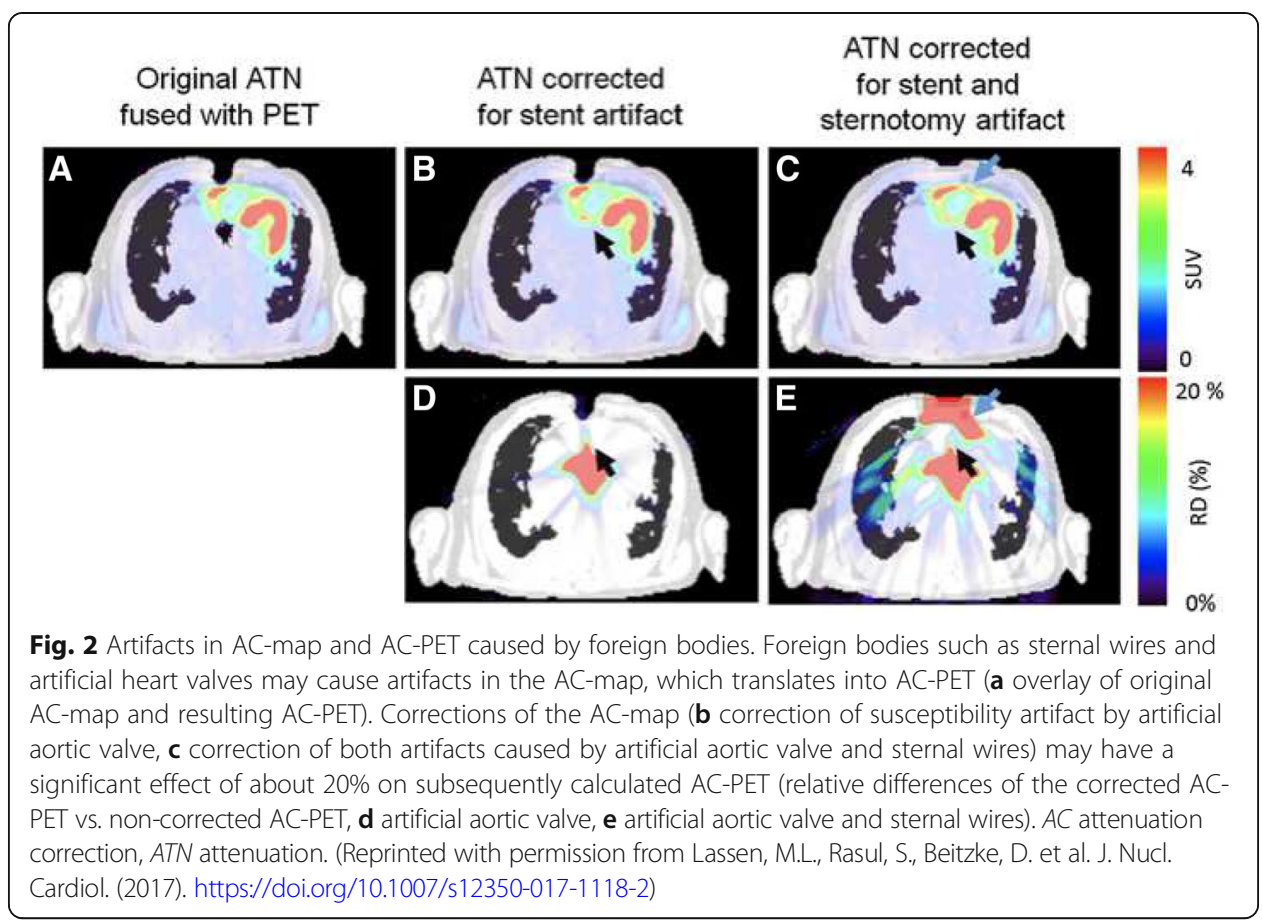

polar maps. There are a handful of software packages (e.g., OsiriX, Pixmeo SARL, Geneva, Switzerland; syngo.via, Siemens Healthineers, Erlangen, Germany) that allow fusion and analysis of fused PET and cardiac MRI data. Outside the scope of academic software developments, to the best of our knowledge, no software packages for fully integrated PET/MRI data-also quantitative in nature-is available. An approach for integrated analysis of cardiac PET and MRI data by co-registered polar maps and mutual segmentation of myocardial boundaries has recently been proposed and may allow

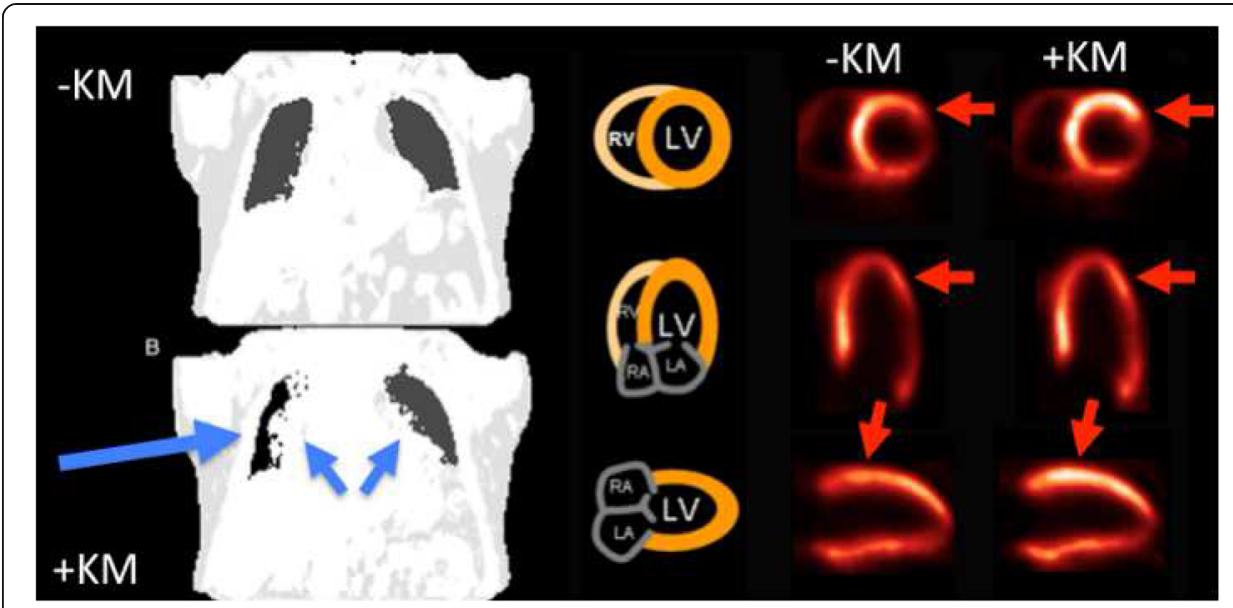

Fig. 3 Effects of contrast media on attenuation-correction in PET/MRI. Attenuation correction (AC)-maps derived from Dixon VIBE MR sequences before (upper row, left) and after contrast media application (bottom row, left). Contrast media application may cause erroneous segmentation of the lungs (right lung: air instead of lung tissue indicated by large blue arrow) and in hilar regions (overestimation of soft tissues bihilarly, small blue arrows). This translates into an erroneous tracer uptake increase on attenuation corrected PET images in the anterolateral wall (red arrows). (Reprinted with permission from Rischpler C, Nekolla SG, PET Clin 11 (2016) 465-477 https://doi.org/10.1016/j.cpet.2016.05.006) 
for robust segmentation and immanent co-registration (Tezgah et al. 2017). Besides the analysis of simultaneously acquired PET/MRI data, most of the mentioned software packages also allow to fuse data from separately acquired PET(/CT) and MRI data. However, this often requires a high amount of user interaction and may result in misregistration. This may particularly be a problem in case of radiotracers that result in weak signals or bind only to a certain part of the heart (e.g., focal inflammation of the myocardium in cardiac sarcoidosis). In these cases, the user has to rely on the (unspecific) uptake of other surrounding structures such as the liver or bones. Another issue is that MRI data is often non-isotropic and that non-axial slices of the body (e.g., short-axis and long-axis of the heart) are acquired. Therefore, volumetric PET data has to be resampled to the respective MRI data. This may be particularly difficult if for example only a certain number of short axis slices were acquired on MRI (e.g., myocardial perfusion with MRI).

\section{Clinical applications}

\section{Myocardial perfusion imaging}

Myocardial perfusion imaging (MPI) using PET or MRI are well-established procedures for the diagnostic work-up of coronary artery disease. PET MPI may be seen as the reference standard for quantitative assessment of myocardial blood flow and coronary flow reserve. Use of the superior characteristics of radiotracers (versus Gd-based contrast agents) and volume coverage of the whole left ventricle, MRI MPI has the advantage that it is performed without any ionizing radiation and has a higher spatial resolution so that even small areas of ischemic or scarred myocardium are detected. Therefore, hybrid PET/MRI may be useful to cross-validate the two techniques with respect to the complex procedure of quantification in MRI. This approach has just been published by Kunze KP et al. (2018). While a good correlation both on slice average and on a segment basis were found, MRI underestimated coronary flow reserve because of an overestimation of resting perfusion, regardless of the applied MRI deconvolution method. One possible synergistic aspect of PET and MRI was also demonstrated in this publication, i.e., the capability of MRI to distinguish subepicardial from subendocardial blood flow. The authors showed one patient with hypertrophic cardiomyopathy, who had an apparent cavity dilation on PET MPI which was caused by subendocardial ischemia as demonstrated by MRI MPI (Fig. 4). One disadvantage is that MRI MPI usually does not cover the whole left ventricle. As the extent of whole left ventricular ischemia is a key factor for therapy guidance (Hachamovitch et al. 2003), the estimation of this parameter may be inaccurate when using only three slices of the left ventricle. While approaches have been undertaken to enable 3D first-pass perfusion MRI for the estimation of myocardial blood flow in the whole left ventricle, this technique is far from being used in clinical routine (Wissmann et al. 2017). Another unfavorable aspect of MRI MPI is the use of Gd-chelate-based contrast agents. These extracellular contrast agents are not taken up by the viable cardiomyocytes and suffer from a low "extraction fraction," which is also not only perfusion-dependent. These factors make quantitative myocardial perfusion assessment using MRI difficult. In contrast, radiotracers with short half-lives are used in PET MPI (the most commonly being O-15 water $\left(T_{1 / 2}=\right.$ $122 \mathrm{~s})$, Rubidium-82 ( $\left.T_{1 / 2}=76 \mathrm{~s}\right)$, and $\mathrm{N}-13$ ammonia $\left(T_{1 / 2}: 10 \mathrm{~min}\right)$ ). While absolute 


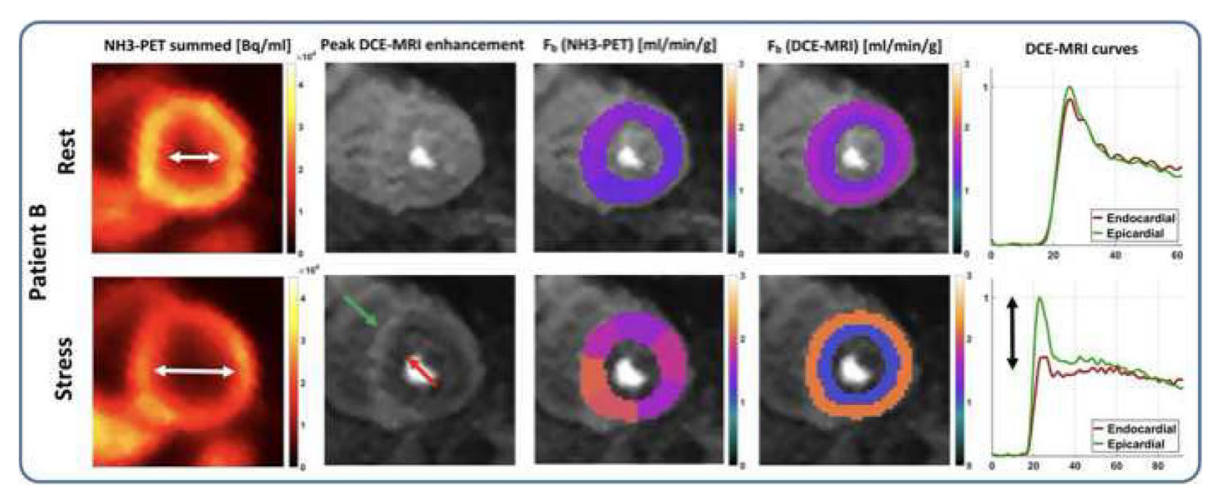

Fig. 4 Hybrid PET/MRI myocardial perfusion assessment in a patient with hypertrophic cardiomyopathy. The apparent dilatation of left ventricular cavity during adenosine challenge (first column) is caused by a stressinduced subendocardial hypoperfusion as demonstrated by MRI MPI (column two to five). (Reprinted with permission from Kunze KP, Nekolla SG, Rischpler C, et al. Myocardial perfusion quantification using simultaneously acquired 13NH3-ammonia PET and dynamic contrast-enhanced MRI in patients at rest and stress. Magn Reson Med. 2018;00:1-14. https://doi.org/10.1002/mrm.27213)

blood flow quantification is routinely performed with these tracers in clinical routine in specialized centers, they have the disadvantage that an on-site cyclotron is a prerequisite or that a cost-intensive generator has to be purchased every month. These factors have prevented PET MPI to be a widely available technique. While novel ${ }^{18} \mathrm{~F}$-labeled perfusion radiotracers are being developed-F-18 Flurpiridaz is the most prominent one-these radiotracers are still evaluated in clinical phase 3 trials (Rischpler et al. 2012; Berman et al. 2013). However, these radiotracers may make PET MPI with absolute flow quantification a more widely available method.

In the future, we expect that hybrid PET/MRI will remain an attractive tool for cross-validation of novel myocardial perfusion approaches, including novel sequences, novel quantification algorithms, novel contrast agents, and/or novel PET radiotracers.

\section{Imaging of myocardial viability}

Repetitive or chronic ischemia (i.e., continuous hypoperfusion) may cause myocardial wall motion abnormalities and a reduced left ventricular pump function. In such a condition, it is known that cardiomyocytes primarily metabolize glucose instead of free fatty acids and that viability of cardiomyocytes is maintained by a protective gene and protein program. This state of contractile dysfunction, however, may be reversible when normal perfusion is reestablished (Rahimtoola 1985) and is therefore often referred to as "hibernating myocardium" (Heusch 1998; Heusch et al. 2005). It is a well-known fact that the presence of hibernating myocardium is associated with poor outcome if not revascularized (Beanlands et al. 1998) and that the extent of hibernating myocardium may serve as an important marker for therapy guidance (Di Carli et al. 1994; D'Egidio et al. 2009; Allman et al. 2002). There are several methods to non-invasively assess myocardial viability (e.g., dobutamine stress echocardiography and MRI, Thallium-201 SPECT), with F-18 FDG PET and late gadolinium enhancement (LGE) MRI most often used (Tillisch et al. 1986). For FDG PET, the patient's heart must be prepared in a way that glucose is the primary substrate and FDG uptake is thus increased. There are several methods to increase the expression of glucose transporters on the cell membrane of cardiomyocytes with oral 
glucose loading, and the hyperinsulinemic-euglycemic clamp technique is most often used. It is recommended to combine FDG PET with a perfusion study (e.g., N-13 ammonia PET or Tc-99 m sestamibi SPECT) in order to identify hibernating myocardium (mismatch between decreased perfusion and increased glucose consumption). Based on a meta-analysis, FDG PET has a 92\% sensitivity among viability imaging approaches and an acceptable specificity of 63\% (Schinkel et al. 2007). LGE MRI is often used as an alternative for viability imaging, even though the term viability is entirely misleading since scarred tissue is actually imaged. The theory behind this approach is that dysfunctional myocardium is more likely to recover the higher the subendocardial proportion of non-scarred (= viable) myocardium. The technique makes use of the fact that Gd-chelate-based contrast agents have a reduced wash-out from areas with increased extravascular space (such as the fibrotic tissue in a myocardial scar) in comparison to a fast wash-out from "healthy" non-scarred myocardium (Klein et al. 2004; Klein et al. 2007). Even though the approaches are quite different (molecular imaging of an increased glucose consumption in viable cells vs. extracellular contrast agent accumulation in fibrotic tissue), a good agreement regarding myocardial viability assessment has been demonstrated (Klein et al. 2002). Benefits of LGE MRI over FDG PET include the lack of ionizing radiation, the highly reproducible accumulation of Gd-based contrast agents in scar tissue, independently from the metabolic state of the patient (which may be an important factor in certain patients such as diabetics), and the high in-plane resolution of MRI of about 1-3 mm, which may permit the distinction between thinned myocardium and scarring (e.g., in the case of a reduced FDG uptake) or the depiction of tiny areas of myocardial scar, which are also known to carry prognostic significance even in patients without overt myocardial infarction (Kim et al. 2000; Kwong et al. 2006). Studies on viability imaging using hybrid FDG PET/MRI are still scarce. In the three available studies, agreements between FDG PET and MRI were reported as moderate to substantial (Fig. 5) (Priamo et al. 2017; Rischpler et al. 2015; Nensa et al. 2013). In one study with a very small patient cohort, adding FDG PET to LGE MRI resulted in a reclassification of about 19\% of segments (Priamo et al. 2017). However, no follow-up to validate the actual impact in terms of wall motion recovery was available.

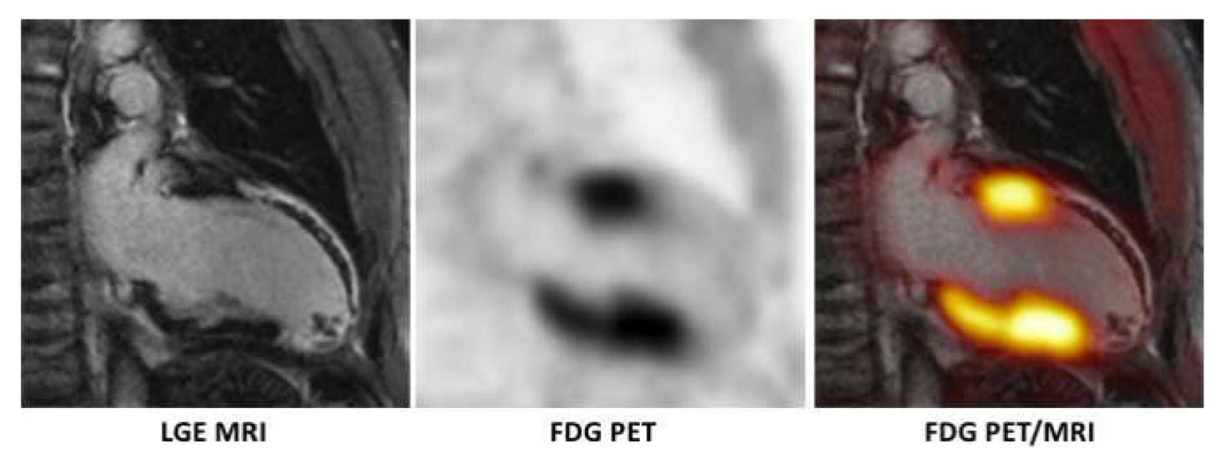

Fig. 5 Viability assessment with hybrid FDG PET/MRI. A FDG PET/MRI (viability protocol with glucose loading) was performed in a mid-aged man early after acute ST-elevation myocardial infarction with occlusion of the left anterior descending (LAD) coronary artery. The inferior wall and the basal part of the anterior wall demonstrate no late gadolinium enhancement (LGE) and a normal FDG uptake and are therefore PET/MRI vital. The remaining parts of the heart in this two-chamber-view demonstrate transmural LGE with subendocardial microvascular obstruction in the anterior wall and almost absent FDG uptake and are therefore judged PET/MRI non-vital 
While this latter study was performed prior to elective revascularization for coronary artery disease, the other two studies were performed early after myocardial infarction. In one study, hybrid FDG PET/MRI was performed early after myocardial infarction in order to compare LGE MRI and FDG PET in dysfunctional myocardium (Rischpler et al. 2015). The aim was to investigate the functional recovery of the dysfunctional segments after 6 months. A high intermethod agreement for the transmurality of LGE and the reduced FDG uptake in the affected segments was found $(K=0.65)$. The wall motion of both the "PET viable" (segments without or only mild decrease in FDG uptake) and the "MRI viable" (non-transmural LGE $<50 \%$ of the myocardial wall) dysfunctional segments was more likely to recover after 6 months. A novel finding was segments which demonstrated a "discordant" pattern of LGE transmurality and FDG uptake, i.e., a reduced FDG uptake but non-transmural or even only minor LGE. Importantly, only around $40 \%$ of these "discordant" segments demonstrated an improvement in wall motion after 6 months. In a study by Nensa et al., the alteration of glucose metabolism in the area of infarction was set in relation to the actual infarct size (assessed by LGE MRI) and to the area at risk (determined by the so-called ESA (endocardial surface area) method) (Nensa et al. 2015). The ESA method defines all myocardial segments as area at risk that demonstrate at least some degree of LGE regardless of the transmurality of LGE. As expected, it was found that the area at risk was larger than the infarct area $(31 \pm 11 \%$ vs $10 \pm 10 \%)$. The most interesting finding was that the area at risk correlated with the extent of altered glucose metabolism indicating that post-ischemic myocardium demonstrates reduced FDG uptake. Still, the pathophysiological background of such metabolic alteration of the myocardium remains unclear and warrants to be further investigated. Until today, there is no study available that investigated the value of integrated viability FDG PET/MRI in patients prior to revascularization with a follow-up examination in order to estimate the predictive value of the combined approach in this setting.

\section{Imaging of focal inflammation}

Using MRI, several processes which may reflect inflammatory processes can be detected. Among those are myocardial fibrosis (accessible using LGE or T1 mapping) and myocardial edema (T2-weighted sequences) (Heusch et al. 2015). Also, pericardial effusion and wall motion abnormalities can be detected with high sensitivity. However, in recent years, FDG PET is increasingly used for the assessment of inflammatory processes in the heart (Erba et al. 2013). FDG PET allows to detect and monitor the extension and grade of the respective inflammatory process which, in turn, allows to distinguish between florid and healed inflammatory processes. As described above, FDG may also be taken up by vital cardiomyocytes when the heart's metabolism runs on carbohydrates. Consequently, an imperative prerequisite for FDG imaging of inflammation is a switch of myocardial metabolism toward the oxidation of free fatty acids. Common patient preparation protocols for this purpose include a high-fat low-carb diet on the day prior to the scan, a prolonged fasting period (e.g., $>12 \mathrm{~h}$ ), and the injection of heparin before the administration of FDG (Nensa et al. 2017; Scholtens et al. 2016). With such protocol, glucose uptake in healthy, non-inflamed myocardium is decreased to a minimum level, and FDG uptake consequently reflects pathological 
processes, such inflammation. This approach may fail, particularly in non-compliant patients, in diabetics and in patients on certain medications such as glucocorticoids, emphasizing an unmet clinical need for PET radiotracers specifically targeting inflammatory processes. Some studies indicate that the chemokine receptor 4 (CXCR4), which may be targeted with high affinity and specificity using Ga-68 pentixafor, is a promising target (Rischpler et al. 2016a). However, CXCR4 is a very complex target which is highly variable and dynamic and is expressed on various cells, including leukocytes, sprouting vessels, and cancer cells. Also, F-18 NaF has been identified as a potential radiotracer to detect plaque vulnerability or to image scar tissue after myocardial infarction, even though the process of tracer accumulation is not completely understood (Marchesseau et al. 2018).

\section{Myocarditis}

Myocarditis is an inflammatory disease of the heart that may be caused by various noxae including microbial infection, hypersensitivity reactions, or autoimmunological diseases. Still, the reference standard for the diagnosis is endomyocardial biopsy with the disadvantages of invasiveness and a high rate of complications. Another problem of endomyocardial biopsy is that sampling errors may occur, as specimens may be taken from not affected parts of the myocardium. This in turn may result in discrepancies between imaging and biopsy results. Consequently, the implementation rate of endomyocardial biopsy is variable between different centers. Another consequence is that non-invasive imaging, particularly MRI, has seen a great increase over the last years for this indication and that clinical decision and therapy is often guided by this imaging modality. The procedure of the MRI scan and its reporting has been standardized by the "Lake Louise Criteria." The imaging protocol includes the minimum of hyperemia (early gadolinium enhancement), edema (T2-weighted imaging), and necrosis/scar (late gadolinium enhancement) imaging, resulting in rather sobering numbers for sensitivity, specificity, and negative predictive value of $67 \%, 91 \%$, and $69 \%$, respectively, when using endomyocardial biopsy as the gold standard (Friedrich et al. 2009). A drawback is that MRI primarily depicts morphological changes of tissue damage but the activity of the inflammatory processes is assessed only indirectly. The combination of PET with MRI may contribute to the solution of this problem. Following initial positive case reports on the use of hybrid FDG PET/MRI for diagnosing, grading, and monitoring myocarditis (Nensa et al. 2014; von Olshausen et al. 2014; Piriou et al. 2015), Nensa et al. published the first prospective study in 65 patients on this matter (Nensa et al. 2018b). In comparison to a variant of the "Lake Louise Criteria" as reference, FDG PET had a sensitivity of $74 \%$ and a specificity of $97 \%$. Most importantly, one patient with biopsy-proven myocarditis had pathological myocardial FDG uptake in the absence of abnormalities in MRI, possibly representing an early stage of the disease, yet without morphological changes of the heart. Another recent publication, however with a very small number of patients, also demonstrated the feasibility of this approach (Hanneman et al. 2017). An open question remains, however, whether or not combined FDG PET/MRI results in an improved diagnosis and patient outcome. An example of a patient with myocarditis who was imaged on a hybrid PET/MRI is depicted in Fig. 6 . 


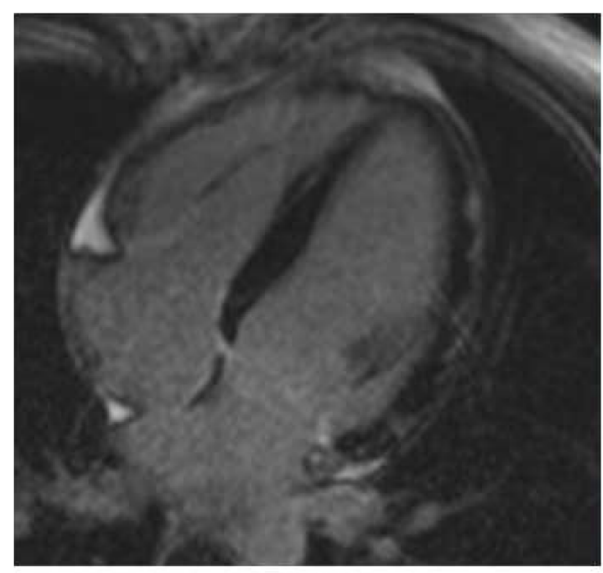

LGE MRI

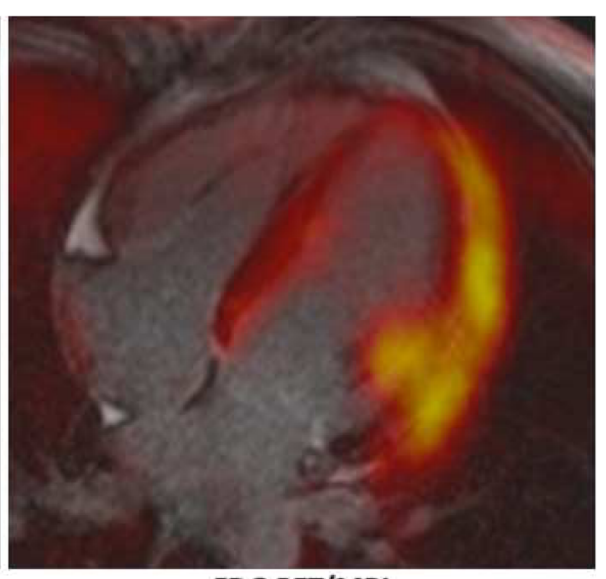

FDG PET/MRI

Fig. 6 FDG PET/MRI in a case of myocarditis. Subepicardial, mostly band-shaped late gadolinium enhancement (LGE) on MRI in the lateral wall of the left ventricle. The FDG uptake on PET clearly exceeds LGE MRI indicating active infection exceeding the scarred areas

\section{Cardiac sarcoidosis}

Another inflammatory disease where hybrid PET/MRI may play a pivotal role is the cardiac involvement in sarcoidosis. In up to $40 \%$ of cases, the initial clinical manifestation of this insidious disease is sudden cardiac death (Sekiguchi et al. 1980). In case of cardiac involvement, the myocardium is infiltrated with inflammatory granulomas which may result in scarring, malignant arrhythmia, and heart failure. It is obvious that cardiac involvement in sarcoidosis must be ruled out reliably. Usually, the guidelines of the Japanese Ministry of Health and Welfare are used to estimate the probability of cardiac involvement. However, these guidelines only recommend cardiac MRI or Ga-67 scintigraphy as imaging tests and not FDG PET. Accumulating evidence suggests a valuable and important role of FDG PET regarding the diagnosis, monitoring, and therapy guidance of cardiac sarcoidosis and an expert consensus clearly recommended this method (Birnie et al. 2014; Slart et al. 2018). A myocardial perfusion study (such as N-13 ammonia PET) may add to the FDG PET for the differentiation between early stage, advanced stage, or healed cardiac sarcoidosis, and also have prognostic implications (Blankstein et al. 2014). In principle, FDG PET may be combined with MRI perfusion. MRI MPI, however, only images a certain number of slices (most often three). There are approaches to perform MRI-based MPI of the entire left ventricle, but this is not implemented in clinical routine yet (Wissmann et al. 2017). Therefore, certain parts of the heart (such as the apex) may not be adequately assessed with a MRI MPI/FDG PET approach. The combination of LGE MRI with FDG PET may have an additional benefit, in the exact illustration of myocardial scarring which is important in the case of ICD implantation (Schneider et al. 2014). While there are some case reports and smaller studies on hybrid FDG PET/MRI for cardiac sarcoidosis, a prospective trial with larger patient numbers is still missing (Hanneman et al. 2017; Schneider et al. 2014; Wada et al. 2016; Kiko et al. 2018; Wicks et al. 2018). An alternative approach may be the assessment of the inflammatory granulomas in the myocardium using radiotracers targeting the somatostatin receptor (e.g., Ga-68 DOTANOC). While first reports using this approach are available for PET/CT, there is no clinical experience with simultaneous PET/MRI (Slart et al. 2017; Gormsen et al. 2016). 
Imaging post-ischemic inflammatory processes in the myocardium

There is an intense inflammatory process in the myocardium after acute myocardial infarction which is orchestrated by different immune cells such as neutrophils and monocytes/macrophages (van der Laan et al. 2012). This inflammatory response is a key factor regarding the development of heart failure after myocardial infarction. In addition to established prognostic factors such as infarct size and ejection fraction, inflammation came into the focus of cardiovascular research in recent years (van der Laan et al. 2012; Curley et al. 2018; Heusch et al. 2014). Lee et al. recently demonstrated in a rodent model of myocardial infarction that the invasion of the myocardium by monocytes can be imaged using FDG PET/MRI (Lee et al. 2012). This imaging approach was subsequently translated into a clinical setting (Wollenweber et al. 2014), and a relation between the post-ischemic myocardial inflammation and the FDG uptake in spleen and bone marrow (as a marker of systemic inflammation) was suggested. Indeed, post-ischemic FDG uptake in the heart is a prognostic marker for left ventricular remodeling after myocardial infarction, independently of infarct size assessed by LGE MRI (Fig. 7) (Rischpler et al. 2016b). Consequently, FDG PET/MRI may be a very promising tool for risk stratification of patients after myocardial infarction regarding the development of heart failure, and it may serve to investigate effects of (novel) interventions with the aim to modulate this inflammatory response.

\section{Conclusion}

Since our first review on cardiac PET/MRI in 2013 (at a time when not a single publication on integrated cardiovascular PET/MRI was available), users have gained more experience with this modality, and the number of potential applications has increased. For myocardial perfusion imaging, hybrid PET/MRI remains mainly a research tool for cross-validation of PET vs. MRI and for the development of new sequences, tracers, and contrast agents. For viability imaging, first publications indicate a possible synergistic effect of FDG PET and cardiac MRI. For inflammatory focus imaging, particularly sarcoidosis and myocarditis, accumulating evidence suggests a clear benefit of this modality. In fact, in the institutions of the review's authors, myocarditis and sarcoidosis are primarily examined using FDG PET/MRI (with suppressed myocardial glucose metabolism). For research questions, PET/MRI has the advantage that biosignals (e.g.,

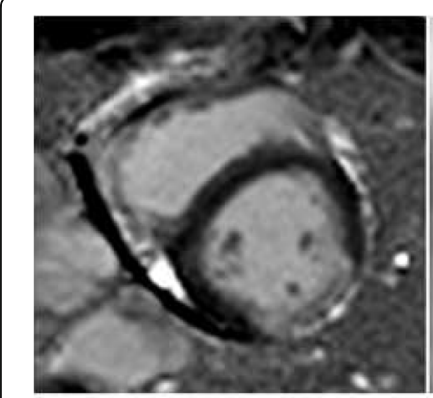

LGE MRI

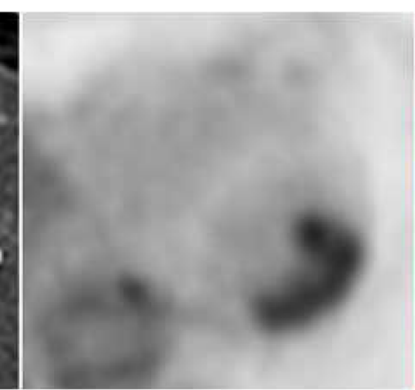

FDG PET

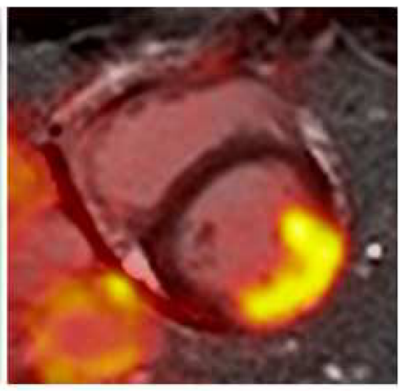

FDG PET/MRI

Fig. 7 Hybrid ${ }^{18}$ F-FDG PET/MRI (suppressed myocardial glucose metabolism) early after ST-elevation myocardial infarction. LGE MRI (left panel) demonstrates myocardial infarction scar of the lateral wall. On FDG PET (middle panel), an intense FDG uptake matching the scar (right panel) is observed. The glucose metabolism in the scar indicates a florid inflammatory response after myocardial infarction 
using novel tracers) can be derived from PET, while MRI provides information on left ventricular pump function and morphology of the heart. In a review from 2016 on the utilization of PET/MRI in 39 sites, most centers expected an increase in cardiovascular utilization. Also, in a recent workshop at the annual meeting of the Society of Nuclear Medicine and Molecular Imaging at Philadelphia (USA) in 2018 with nine experienced PET/MRI centers, most users expected a growing number of cardiovascular PET/MRI studies. In conclusion, cardiovascular PET/MRI has arrived in clinical routine and has gained appreciation in the cardiovascular community.

\begin{abstract}
Abbreviations
AC: Attenuation correction; APD: Avalanche photodiodes; CAIPIRINHA: Controlled aliasing in parallel imaging results in higher acceleration; CT: Computed tomography; CXCR4: Chemokine receptor 4; ESA: Endocardial surface area; FDG: Fludeoxyglucose; HUGE: $B_{0}$ homogenization using gradient enhancement; LGE: Late gadolinium enhancement; MLAA: Maximum likelihood estimation of activity and attenuation; MPI: Myocardial perfusion imaging; MRI: Magnetic resonance imaging; PET: Positron emission tomography; SiPM: Silicon photomultiplier; SPECT: Single-photon emission computed tomography; SPGR: Spoiled gradient recalled echo; SUV: Standardized uptake value
\end{abstract}

\title{
Acknowledgements
}

Not applicable.

Funding

Not applicable.

Availability of data and materials

Not applicable.

\section{Authors' contributions}

All authors contributed significantly to this article. CR, SGN, GH, LU, TR, PH, KH, and FN have participated in drafting the article, have revisited it critically, and have given final approval to this article.

Ethics approval and consent to participate

Not applicable.

\section{Competing interests}

The authors declare that they have no competing interests.

\section{Publisher's Note}

Springer Nature remains neutral with regard to jurisdictional claims in published maps and institutional affiliations.

\footnotetext{
Author details

${ }^{1}$ Department of Nuclear Medicine, University Hospital Essen, University of Duisburg-Essen, Essen, Germany. ${ }^{2}$ Nuklearmedizinische Klinik und Poliklinik, Klinikum rechts der Isar, Technische Universität München, Ismaninger Straße 22, 81675 Munich, Germany. ${ }^{3}$ DZHK (Deutsches Zentrum für Herz-Kreislauf-Forschung e.V.) partner site Munich Heart alliance, Munich, Germany. ${ }^{4}$ Institute for Pathophysiology, University Hospital Essen, University of Duisburg-Essen, Essen, Germany. ${ }^{5}$ Department of Diagnostic and Interventional Radiology and Neuroradiology, University Hospital Essen, University of Duisburg-Essen, Essen, Germany. ${ }^{6}$ Department of Cardiology and Vascular Medicine, University Hospital Essen, West German Heart and Vascular Center, University of Duisburg-Essen, Essen, Germany. ${ }^{7}$ Department of Diagnostic and Interventional Radiology, Medical Faculty, University Düsseldorf, Düsseldorf, Germany.
}

Received: 27 August 2018 Accepted: 17 December 2018

Published online: 22 January 2019

\section{References}

Allman KC, Shaw L, Hachamovitch R, Udelson JE (2002) Myocardial viability testing and impact of revascularization on prognosis in patients with coronary artery disease and left ventricular dysfunction: a meta-analysis. J Am Coll Cardiol 39(7):1151-1158

Beanlands RS, Hendry PJ, Masters RG, deKemp RA, Woodend K, Ruddy TD (1998) Delay in revascularization is associated with increased mortality rate in patients with severe left ventricular dysfunction and viable myocardium on fluorine 18fluorodeoxyglucose positron emission tomography imaging. Circulation 98(19 Suppl):|I51-||156

Berman DS, Maddahi J, Tamarappoo BK, Czernin J, Taillefer R, Udelson JE et al (2013) Phase II safety and clinical comparison with single-photon emission computed tomography myocardial perfusion imaging for detection of coronary artery disease: flurpiridaz F 18 positron emission tomography. J Am Coll Cardiol 61(4):469-477

Beyer T, Lassen ML, Boellaard R, Delso G, Yaqub M, Sattler B et al (2016) Investigating the state-of-the-art in whole-body MRbased attenuation correction: an intra-individual, inter-system, inventory study on three clinical PET/MR systems. MAGMA 29(1):75-87 
Birnie DH, Sauer WH, Bogun F, Cooper JM, Culver DA, Duvernoy CS et al (2014) HRS expert consensus statement on the diagnosis and management of arrhythmias associated with cardiac sarcoidosis. Heart Rhythm 11(7):1305-1323

Blankstein R, Osborne M, Naya M, Waller A, Kim CK, Murthy VL et al (2014) Cardiac positron emission tomography enhances prognostic assessments of patients with suspected cardiac sarcoidosis. J Am Coll Cardiol 63(4):329-336

Blumhagen JO, Braun H, Ladebeck R, Fenchel M, Faul D, Scheffler K et al (2014) Field of view extension and truncation correction for MR-based human attenuation correction in simultaneous MR/PET imaging. Med Phys 41(2):022303

Coombs BD, Szumowski J, Coshow W (1997) Two-point Dixon technique for water-fat signal decomposition with B0 inhomogeneity correction. Magne Reson Med 38(6):884-889

Curley D, Lavin Plaza B, Shah AM, Botnar RM (2018) Molecular imaging of cardiac remodelling after myocardial infarction. Basic Res Cardiol 113(2):10

D'Egidio G, Nichol G, Williams KA, Guo A, Garrard L, deKemp R et al (2009) Increasing benefit from revascularization is associated with increasing amounts of myocardial hibernation: a substudy of the PARR-2 trial. J Am Coll Cardiol Img 2(9): 1060-1068

Delso G, Furst S, Jakoby B, Ladebeck R, Ganter C, Nekolla SG et al (2011) Performance measurements of the Siemens mMR integrated whole-body PET/MR scanner. J Nucl Med 52(12):1914-1922

Di Carli MF, Davidson M, Little R, Khanna S, Mody FV, Brunken RC et al (1994) Value of metabolic imaging with positron emission tomography for evaluating prognosis in patients with coronary artery disease and left ventricular dysfunction. Am J Cardiol 73(8):527-533

Erba PA, Sollini M, Lazzeri E, Mariani G (2013) FDG-PET in cardiac infections. Semin Nucl Med 43(5):377-395

Freitag MT, Fenchel M, Baumer P, Heusser T, Rank CM, Kachelriess M et al (2017) Improved clinical workflow for simultaneous whole-body PET/MRI using high-resolution CAIPIRINHA-accelerated MR-based attenuation correction. Eur J Radiol 96:12-20

Friedrich MG, Sechtem U, Schulz-Menger J, Holmvang G, Alakija P, Cooper LT et al (2009) Cardiovascular magnetic resonance in myocarditis: a JACC white paper. J Am Coll Cardiol 53(17):1475-1487

Fürst S, Souvatzoglu M, Rischpler C, Ziegler S, Schwaiger M, Nekolla S (2012) Effects of MR contrast agents on attenuation map generation and cardiac PET quantification in PET/MR. J Nucl Med 53(Supplement 1):139

Gormsen LC, Haraldsen A, Kramer S, Dias AH, Kim WY, Borghammer P (2016) A dual tracer (68)Ga-DOTANOC PET/CT and (18)F-FDG PET/CT pilot study for detection of cardiac sarcoidosis. EJNMMI Res 6(1):52

Hachamovitch R, Hayes SW, Friedman JD, Cohen I, Berman DS (2003) Comparison of the short-term survival benefit associated with revascularization compared with medical therapy in patients with no prior coronary artery disease undergoing stress myocardial perfusion single photon emission computed tomography. Circulation 107(23):2900-2907

Hanneman K, Kadoch M, Guo HH, Jamali M, Quon A, lagaru A et al (2017) Initial experience with simultaneous 18F-FDG PET/ MRI in the evaluation of cardiac sarcoidosis and myocarditis. Clin Nucl Med 42(7):e328-ee34

Heusch G (1998) Hibernating myocardium. Physiol Rev 78(4):1055-1085

Heusch G, Libby P, Gersh B, Yellon D, Bohm M, Lopaschuk G et al (2014) Cardiovascular remodelling in coronary artery disease and heart failure. Lancet 383(9932):1933-1943

Heusch G, Schulz R, Rahimtoola SH (2005) Myocardial hibernation: a delicate balance. Am J Physiol Heart Circ Physiol 288(3): H984-H999

Heusch P, Nensa F, Heusch G (2015) Is MRI really the gold standard for the quantification of salvage from myocardial infarction? Circ Res 117(3):222-224

Huang SC, Carson RE, Phelps ME, Hoffman EJ, Schelbert HR, Kuhl DE (1981) A boundary method for attenuation correction in positron computed tomography. J Nucl Med 22(7):627-637

Jimenez-Ballve A, Perez-Castejon MJ, Delgado-Bolton RC, Sanchez-Enrique C, Vilacosta I, Vivas D et al (2016) Assessment of the diagnostic accuracy of (18)F-FDG PET/CT in prosthetic infective endocarditis and cardiac implantable electronic device infection: comparison of different interpretation criteria. Eur J Nucl Med Mol Imaging 43(13):2401-2412

Kiko T, Masuda A, Nemoto A, Tominaga H, Oriuchi N, Yoshihisa A et al (2018) Cardiac sarcoidosis after glucocorticoid therapy evaluated by (18)F-fluorodeoxyglucose PET/MRI. J Nucl Cardiol 25(2):685-687

Kim RJ, Wu E, Rafael A, Chen EL, Parker MA, Simonetti O et al (2000) The use of contrast-enhanced magnetic resonance imaging to identify reversible myocardial dysfunction. N Engl J Med 343(20):1445-1453

Klein C, Nekolla SG, Balbach T, Schnackenburg B, Nagel E, Fleck E et al (2004) The influence of myocardial blood flow and volume of distribution on late Gd-DTPA kinetics in ischemic heart failure. J Magn Reson Imaging 20(4):588-593

Klein C, Nekolla SG, Bengel FM, Momose M, Sammer A, Haas F et al (2002) Assessment of myocardial viability with contrastenhanced magnetic resonance imaging: comparison with positron emission tomography. Circulation 105(2):162-167

Klein C, Schmal TR, Nekolla SG, Schnackenburg B, Fleck E, Nagel E (2007) Mechanism of late gadolinium enhancement in patients with acute myocardial infarction. J Cardiovasc Magn Reson 9(4):653-658

Kunze KP, Nekolla SG, Rischpler C, Zhang SH, Hayes C, Langwieser N et al (2018) Myocardial perfusion quantification using simultaneously acquired (13) NH3 -ammonia PET and dynamic contrast-enhanced MRI in patients at rest and stress. Magn Reson Med. https://doi.org/10.1002/mrm.27213

Kwong RY, Chan AK, Brown KA, Chan CW, Reynolds HG, Tsang S et al (2006) Impact of unrecognized myocardial scar detected by cardiac magnetic resonance imaging on event-free survival in patients presenting with signs or symptoms of coronary artery disease. Circulation 113(23):2733-2743

Lassen ML, Rasul S, Beitzke D, Stelzmuller ME, Cal-Gonzalez J, Hacker M et al (2017) Assessment of attenuation correction for myocardial PET imaging using combined PET/MRI. J Nucl Cardiol. https://doi.org/10.1007/s12350-017-1118-2

Lau JMC, Laforest R, Sotoudeh H, Nie X, Sharma S, McConathy J et al (2017) Evaluation of attenuation correction in cardiac PET using PET/MR. J Nucl Cardiol 24(3):839-846

Lee WW, Marinelli B, van der Laan AM, Sena BF, Gorbatov R, Leuschner F et al (2012) PET/MRI of inflammation in myocardial infarction. J Am Coll Cardiol 59(2):153-163

Levin C, Deller T, Peterson W, Maramraju SH, Kim C, Prost R (2014) Initial results of simultaneous whole-body ToF PET/MR. J Nucl Med 55(Supplement 1):660

Levin CS, Maramraju SH, Khalighi MM, Deller TW, Delso G, Jansen F (2016) Design features and mutual compatibility studies of the time-of-flight PET capable GE SIGNA PET/MR system. IEEE Trans Med Imaging 35: 1907-1914 
Lindemann ME, Oehmigen M, Blumhagen JO, Gratz M, Quick HH (2017) MR-based truncation and attenuation correction in integrated PET/MR hybrid imaging using HUGE with continuous table motion. Med Phys 44(9):4559-4572

Marchesseau S, Seneviratna A, Sjoholm AT, Qin DL, JXM H, Hausenloy DJ et al (2018) Hybrid PET/CT and PET/MRI imaging of vulnerable coronary plaque and myocardial scar tissue in acute myocardial infarction. J Nucl Cardiol 25(6):2001-2011. https://doi.org/10.1007/s12350-017-0918-8. Epub 2017 May 12.

Martinez-Moller A, Souvatzoglou M, Delso G, Bundschuh RA, Chefd'hotel C, Ziegler Sl et al (2009) Tissue classification as a potential approach for attenuation correction in whole-body PET/MRI: evaluation with PET/CT data. J Nucl Med 50(4):520-526

Nensa F, Bamberg F, Rischpler C, Menezes L, Poeppel TD, la Fougere C et al (2018a) Hybrid cardiac imaging using PET/MRI: a joint position statement by the European Society of Cardiovascular Radiology (ESCR) and the European Association of Nuclear Medicine (EANM). Eur Radiol 28(10):4086-4101

Nensa F, Kloth J, Tezgah E, Poeppel TD, Heusch P, Goebel J et al (2018b) Feasibility of FDG-PET in myocarditis: comparison to CMR using integrated PET/MRI. J Nucl Cardiol 25(3):785-794

Nensa F, Poeppel T, Tezgah E, Heusch P, Nassenstein K, Mahabadi AA et al (2015) Integrated FDG PET/MR imaging for the assessment of myocardial salvage in Reperfused acute myocardial infarction. Radiology 276(2):400-407

Nensa F, Poeppel TD, Beiderwellen K, Schelhorn J, Mahabadi AA, Erbel R et al (2013) Hybrid PET/MR imaging of the heart: feasibility and initial results. Radiology 268(2):366-373

Nensa F, Poeppel TD, Krings P, Schlosser T (2014) Multiparametric assessment of myocarditis using simultaneous positron emission tomography/magnetic resonance imaging. Eur Heart J 35(32):2173

Nensa F, Tezgah E, Schweins K, Goebel J, Heusch P, Nassenstein K et al (2017) Evaluation of a low-carbohydrate diet-based preparation protocol without fasting for cardiac PET/MR imaging. J Nucl Cardiol 24(3):980-988

Nuyts J, Bal G, Kehren F, Fenchel M, Michel C, Watson C (2012) Completion of a truncated attenuation image from the attenuated PET emission data. IEEE Trans Med Imaging 2013;32(2):237-46. https://doi.org/10.1109/TMI.2012.2220376. Epub 2012 Sep 21

Nuyts J, Dupont P, Stroobants S, Bennick R, Mortelmans L, Suetens P (1999) Simultaneous maximum a posteriori reconstruction of attenuation and activity distributions from emission sinograms. IEEE Trans Med Imaging 18(5):393-403

Paulus DH, Quick HH, Geppert C, Fenchel M, Zhan Y, Hermosillo G et al (2015) Whole-body PET/MR imaging: quantitative evaluation of a novel model-based MR attenuation correction method including bone. J Nucl Med. 56(7):1061-1066

Pichler BJ, Judenhofer MS, Catana C, Walton JH, Kneilling M, Nutt RE et al (2006) Performance test of an LSO-APD detector in a 7-T MRI scanner for simultaneous PET/MRI. J Nucl Med. 47(4):639-647

Piriou N, Sassier J, Pallardy A, Serfaty JM, Trochu JN (2015) Utility of cardiac FDG-PET imaging coupled to magnetic resonance for the management of an acute myocarditis with non-informative endomyocardial biopsy. Eur Heart I Cardiovasc Imaging 16(5):574

Priamo J, Adamopoulos D, Rager O, Frei A, Noble S, Carballo D et al (2017) Downstream indication to revascularization following hybrid cardiac PET/MRI: preliminary results. Nucl Med Commun 38(6):515-522

Rahimtoola SH (1985) A perspective on the three large multicenter randomized clinical trials of coronary bypass surgery for chronic stable angina. Circulation 72(6 Pt 2):V123-V135

Rischpler C, Dirschinger RJ, Nekolla SG, Kossmann H, Nicolosi S, Hanus F et al (2016b) Prospective evaluation of 18Ffluorodeoxyglucose uptake in Postischemic myocardium by simultaneous positron emission tomography/magnetic resonance imaging as a prognostic marker of functional outcome. Circ Cardiovasc Imaging 9(4):e004316

Rischpler C, Langwieser N, Souvatzoglou M, Batrice A, van Marwick S, Snajberk J et al (2015) PET/MRI early after myocardial infarction: evaluation of viability with late gadolinium enhancement transmurality vs. 18F-FDG uptake. Eur Heart J Cardiovasc Imaging 16(6):661-669

Rischpler C, Nekolla SG, Dregely I, Schwaiger M (2013) Hybrid PET/MR imaging of the heart: potential, initial experiences, and future prospects. J Nucl Med 54(3):402-415

Rischpler C, Nekolla SG, Kossmann H, Dirschinger RJ, Schottelius M, Hyafil F et al (2016a) Upregulated myocardial CXCR4expression after myocardial infarction assessed by simultaneous GA-68 pentixafor PET/MRI. J Nucl Cardiol 23(1):131-133

Rischpler C, Park MJ, Fung GS, Javadi M, Tsui BM, Higuchi T (2012) Advances in PET myocardial perfusion imaging: F-18 labeled tracers. Ann Nucl Med 26(1):1-6

Ruhlmann V, Heusch P, Kuhl H, Beiderwellen K, Antoch G, Forsting M et al (2016) Potential influence of gadolinium contrast on image segmentation in MR-based attenuation correction with Dixon sequences in whole-body 18F-FDG PET/MR. MAGMA 29(2):301-308

Samarin A, Burger C, Wollenweber SD, Crook DW, Burger IA, Schmid DT et al (2012) PET/MR imaging of bone lesions-implications for PET quantification from imperfect attenuation correction. Eur J Nucl Med Mol Imaging 39(7):1154-1160

Schinkel AF, Poldermans D, Elhendy A, Bax JJ (2007) Assessment of myocardial viability in patients with heart failure. J Nucl Med 48(7):1135-1146

Schneider S, Batrice A, Rischpler C, Eiber M, Ibrahim T, Nekolla SG (2014) Utility of multimodal cardiac imaging with PET/MRI in cardiac sarcoidosis: implications for diagnosis, monitoring and treatment. Eur Heart J 35(5):312

Scholtens AM, Verberne HJ, Budde RP, Lam MG (2016) Additional heparin preadministration improves cardiac glucose metabolism suppression over low-carbohydrate diet alone in 18F-FDG PET imaging. J Nucl Med 57(4):568-573

Schulz V, Torres-Espallardo I, Renisch S, Hu Z, Ojha N, Bornert P et al (2011) Automatic, three-segment, MR-based attenuation correction for whole-body PET/MR data. Eur J Nucl Med Mol Imaging 38(1):138-152

Sekiguchi M, Numao Y, Imai M, Furuie T, Mikami R (1980) Clinical and histopathological profile of sarcoidosis of the heart and acute idiopathic myocarditis. Concepts through a study employing endomyocardial biopsy. I. Sarcoidosis. Jpn Circ J 44(4):249-263

Slart R, Glaudemans A, Lancellotti P, Hyafil F, Blankstein R, Schwartz RG et al (2018) A joint procedural position statement on imaging in cardiac sarcoidosis: from the cardiovascular and Inflammation \& Infection Committees of the European Association of Nuclear Medicine, the European Association of Cardiovascular Imaging, and the American society of nuclear cardiology. J Nucl Cardiol 25(1):298-319

Slart R, Koopmans KP, van Geel PP, Kramer H, Groen HJM, Gan CT et al (2017) Somatostatin receptor based hybrid imaging in sarcoidosis. Eur J Hybrid Imaging 1(1):7

Nensa FTDP, Tezgah E, Heusch P, Nassenstein K, Forsting M et al (2017) Integrated assessment of cardiac PET/MRI: coregistered PET and MRI polar plots by mutual MR-based segmentation of the left ventricular myocardium. World J Cardiovasc Dis 7:91-104 
Tillisch J, Brunken R, Marshall R, Schwaiger M, Mandelkern M, Phelps M et al (1986) Reversibility of cardiac wall-motion abnormalities predicted by positron tomography. N Engl J Med 314(14):884-888

van der Laan AM, Nahrendorf M, Piek JJ (2012) Healing and adverse remodelling after acute myocardial infarction: role of the cellular immune response. Heart 98(18):1384-1390

von Olshausen G, Hyafil F, Langwieser N, Laugwitz KL, Schwaiger M, Ibrahim T (2014) Detection of acute inflammatory myocarditis in Epstein Barr virus infection using hybrid 18F-fluoro-deoxyglucose-positron emission tomography/magnetic resonance imaging. Circulation 130(11):925-926

Wada K, Niitsuma T, Yamaki T, Masuda A, Ito H, Kubo H et al (2016) Simultaneous cardiac imaging to detect inflammation and scar tissue with (18)F-fluorodeoxyglucose PET/MRI in cardiac sarcoidosis. J Nucl Cardiol 23(5):1180-1182

Wicks EC, Menezes LJ, Barnes A, Mohiddin SA, Sekhri N, Porter JC et al (2018) Diagnostic accuracy and prognostic value of simultaneous hybrid 18F-fluorodeoxyglucose positron emission tomography/magnetic resonance imaging in cardiac sarcoidosis. Eur Heart J Cardiovasc Imaging 19(7):757-767

Wissmann L, Gotschy A, Santelli C, Tezcan KC, Hamada S, Manka R et al (2017) Analysis of spatiotemporal fidelity in quantitative 3D first-pass perfusion cardiovascular magnetic resonance. J Cardiovasc Magn Reson 19(1):11

Wollenweber T, Roentgen P, Schafer A, Schatka I, Zwadlo C, Brunkhorst T et al (2014) Characterizing the inflammatory tissue response to acute myocardial infarction by clinical multimodality noninvasive imaging. Circ Cardiovasc Imaging. 7(5):811-818

Zaidi H, Ojha N, Morich M, Griesmer J, Hu Z, Maniawski P et al (2011) Design and performance evaluation of a whole-body ingenuity TF PET-MRI system. Phys Med Biol 56(10):3091-3106

Submit your manuscript to a SpringerOpen ${ }^{\circ}$ journal and benefit from:

- Convenient online submission

- Rigorous peer review

- Open access: articles freely available online

- High visibility within the field

- Retaining the copyright to your article

Submit your next manuscript at $\boldsymbol{\nabla}$ springeropen.com 